\title{
Implicaciones del consumo y de la flexibilidad de la oferta laboral en el bienestar de los partícipes de planes de pensiones del Sistema de Empleo
}

\author{
FRANCISCO J. PELÁEZ FERMOSO
}

Departamento de Economía Aplicada, UNIVERSIDAD DE VALLADOLID, ESPAÑA. Email: ppelaez@eco.uva.es

\section{ANA GARCÍA GONZÁLEZ}

Departamento de Economía Aplicada, UNIVERSIDAD DE VALLADOLID, ESPAÑA. Email: anagar@eco.uva.es

JESÚS Ma . GÓMEZ GARCÍA

Departamento de Economía Aplicada, UNIVERSIDAD DE VALLADOLID, ESPAÑA. Email: jmgomez@eco.uva.es

\section{RESUMEN}

Este trabajo pretende llevar a cabo un análisis comparativo de los planes de pensiones del sistema de empleo de aportación definida y los de prestación definida desde el punto de vista del bienestar percibido por cada trabajador. En un contexto en el que se considera flexibilidad en la oferta laboral de la empresa promotora del plan de pensiones, se busca maximizar la utilidad que reportan las preferencias temporales de consumo y ocio de cada empleado. Se plantea el problema como uno de optimización dinámica de elección intertemporal, considerando que el comportamiento de los rendimientos de las inversiones de los recursos financieros del fondo del plan y de los tantos anuales de crecimiento de los salarios vienen descritos a través de procesos markovianos de tiempo discreto. Para cada tipo de plan de pensiones, se analizan los valores de consumo y de ocio óptimos que maximizan la utilidad (bienestar) del trabajador, partícipe del plan, en los diferentes períodos del horizonte temporal analizado.

Palabras clave: Modelo de ciclo de vida, planes de pensiones del sistema de empleo, proceso markoviano, programación dinámica.

\section{Implications of Consumption and Labor Supply Flexibility in the Welfare of the Holders of Employment System Pension Plans}

\begin{abstract}
This work aims to carry out a comparative analysis of the pension plans of the employment system (both defined benefit and defined contribution plans) from the point of view of the welfare perceived by each worker. Considering flexibility in the labor supply of the promoting company of the pension scheme, we seek to maximize the utility of the time preferences of consumption and leisure for each employee. We propose a dynamic optimization problem of intertemporal choice, and we describe both the returns on the investments of the Fund and the annual wage growth rates as discrete markovian processes. For each type of pension plan, we analyse the optimal consumption and leisure values that maximize the utility (welfare) of the worker over several periods of time.
\end{abstract}

Keywords: Life Cycle Model, Pension Plans Of Employment System, Markovian Process, Dynamic Programming.

Clasificación JEL: C61, D91, G23

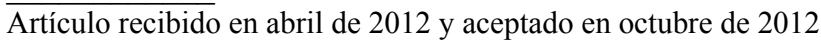

Artículo disponible en versión electrónica en la página www.revista-eea.net, ref. ə-30309 


\section{INTRODUCCIÓN}

Desde un punto de vista técnico, los planes y fondos de pensiones se consideran instrumentos financieros de previsión social de carácter privado y de ahorro a largo plazo que aparecen en el mercado para, en parte, poder subsanar las posibles deficiencias de índole económica y financiera que caracterizan a la mayoría de los sistemas públicos de previsión social existentes, buscando mantener un nivel adquisitivo adecuado de los trabajadores en el momento de abandonar su actividad laboral por medio de la obtención de la máxima rentabilidad de los activos en los que se invierten los recursos disponibles del fondo de pensiones (Alexander y Sharpe, 1989).

Entre las diferentes modalidades de planes y fondos de pensiones que a nivel mundial operan actualmente en el mercado, cabe reseñar los planes de pensiones del Sistema de Empleo, que son aquéllos que promueven las empresas a favor de sus trabajadores con el fin de incentivar su productividad y su permanencia en las mismas (Peña Esteban, 2000; Peláez y García, 2004). Dentro de este tipo de planes analizaremos los planes de pensiones de Aportación Definida (AD) y los de Prestación Definida (PD).

Siguiendo a estos autores, los planes de pensiones de aportación definida se caracterizan porque definen, en primer lugar, la cuantía de la aportación que debe realizar sistemáticamente el promotor del plan a la cuenta de posición que tiene cada partícipe-trabajador en el fondo de pensiones donde aquél se integra. Por tanto, la prestación de jubilación devengada a partir de la edad de jubilación alcanzada por cada partícipe del plan, en el caso de que sobreviva a ésta, viene definida por el montante constituido por las referidas aportaciones y los rendimientos que, en forma de intereses, son producidos por las inversiones de las mismas. En este caso, la fuente de riesgo más relevante para el partícipe-trabajador será el comportamiento de los rendimientos de las inversiones del fondo de pensiones.

Por otro lado, los planes de pensiones de prestación definida se caracterizan porque son las prestaciones de jubilación las que se definen previamente, en el momento de la constitución del plan, teniendo en cuenta el número de años trabajados y del salario final o medio de los últimos años estimado para cada partícipe-trabajador del plan. En este caso, el trabajador asume generalmente un riesgo importante respecto de las prestaciones de jubilación que deberá percibir a partir de esa edad en tanto sobreviva, ya que en estas prestaciones influye la evolución de los salarios y la edad de jubilación, que pueden ser inciertas. Conviene precisar que para la realización de este trabajo se considerará que ambos tipos de planes de pensiones sólo cubren prestaciones a partir de la edad de jubilación del partícipe del plan.

Lo que se pretende través de este estudio, es realizar un análisis comparativo de ambos tipos de planes utilizando la teoría tradicional de la elección intertem- 
poral del consumidor, teniendo en cuenta las posibles combinaciones de consumo y de ocio (horas no trabajadas) de cada trabajador-partícipe del plan de pensiones. Las preferencias respecto del consumo y del ocio de cada trabajador $\mathrm{y}$, por tanto, su bienestar en función de los mismos, están determinadas por una función de utilidad que es aditiva respecto del tiempo. En este contexto, se puede constatar que las relaciones entre el ahorro y los ciclos económicos, el ahorro y el bienestar o el ahorro y la competitividad, han sido objeto de continuos debates. La literatura existente sobre el estudio del consumo y el ahorro es muy extensa (Heckman, 1974; Hubbard, 1987; Deaton, 1995). Decidir cuánto consumir es establecer qué cantidad de dinero se gasta hoy frente a guardarlo para financiar el consumo futuro. Por tanto, la base adecuada para elaborar una teoría del consumo es la teoría de la elección intertemporal en distintos períodos, considerando bien certeza absoluta, o bien incertidumbre. Esta elección viene descrita por medio de un conjunto de preferencias intertemporales recogidas a través de una función de utilidad determinada. Las preferencias intertemporales pueden ser aditivas o fuertemente separables en el horizonte temporal analizado. El trabajador percibe una renta de trabajo (salario) en cada uno de los períodos analizados y ha de decidir qué parte debe consumir y cuál invertir, en aras a maximizar su bienestar o utilidad.

Si se considera un modelo más complejo que el anterior y se introduce la oferta de trabajo de una determinada empresa, que puede ser flexible, es decir, que el número de horas que el trabajador decide trabajar o dedicar al ocio es función del salario establecido, en este caso, tanto el ocio como el consumo aparecen en la restricción presupuestaria que se asocia a la riqueza disponible de cada trabajador. Por tanto, la maximización de la función de utilidad sujeta a la restricción presupuestaria de cada período es el problema principal de la elección intertemporal (Varian, 1998).

Este trabajo se distribuye en diferentes secciones. Después de esta introducción, en la sección 2 se describen los objetivos y la metodología aplicada en el análisis que se llevará a cabo con posterioridad. A continuación, en la sección 3 se realiza una descripción del modelo de optimización intertemporal planteado, de las hipótesis y variables que en él se consideran y se describe, igualmente, el proceso de optimización del problema. En la sección 4 se realiza una simulación del modelo planteado teniendo en cuenta diferentes alternativas para los tantos de rendimiento del fondo de pensiones y de los tantos de crecimiento de los salarios modelados a través de procesos markovianos en tiempo discreto. Finalmente, en la sección 5 se recogen las principales conclusiones extraídas de los resultados de la simulación del modelo asociado al problema de optimización intertemporal planteado y, por último, las referencias bibliográficas utilizadas en el desarrollo de este trabajo. 


\section{OBJETIVOS Y METODOLOGÍA}

En este apartado se describen los objetivos y la metodología aplicada para realizar un análisis comparativo de ambos tipos de planes de pensiones:

Realización de un análisis comparativo de carácter económico-financiero de los planes del Sistema de Empleo de aportación definida (AD) y de prestación definida (PD). Para tal fin, haremos uso de la teoría de la elección del consumidor y del modelo tradicional del ciclo de vida ( Deaton y Muellbauer, 1994; Deaton, 1995).

Planteamiento de un modelo de programación dinámica en tiempo discreto para un horizonte temporal de $T=3$ períodos, resolviendo el mismo como un problema de optimización para cada período (Boyle et al., 1998; Barbolla et al., 2001) y descripción de las hipótesis y variables que caracterizan a dicho modelo.

Utilización de la teoría tradicional de la elección del consumidor, suponiendo que el partícipe-trabajador tiene unas determinadas preferencias respecto del consumo y del ocio y que son representadas a través de una determinada función de utilidad que es logarítmica y aditiva respecto del tiempo (Deaton, 1995; Varian, 2007).

Simulación del modelo para determinar el consumo y el ocio óptimos que maximizan la función de utilidad indirecta de cada período (Varian, 1998), empleando procesos Markovianos de tipo discreto para describir la evolución de algunos de sus parámetros, como los tantos de crecimiento de los salarios y los tantos de rendimientos de las inversiones del fondo del plan de pensiones (Taha, 2007; Hillier y Lieberman, 2010).

\section{EL MODELO DE OPTIMIZACIÓN INTERTEMPORAL}

Teniendo en cuenta los dos tipos de planes de pensiones que acabamos de reseñar y la estructura financiera, características y particularidades de los mismos (Bodie et al., 1988; Cohen, 1990), se analizan ambos tipos de planes de pensiones utilizando un enfoque y metodología característicos. Para ello, se supone que el consumidor, trabajador-partícipe del plan, puede realizar una elección en términos de consumo y ocio, teniendo en cuenta que existe flexibilidad en la oferta de trabajo por parte de la empresa promotora del plan y que es al partícipe del mismo a quien corresponde decidir el número de horas que desea trabajar o dedicar al ocio. En consecuencia, cada partícipe-trabajador del plan tratará de alcanzar el mayor bienestar posible en términos de la utilidad que le reportan el consumo y el ocio durante toda su trayectoria laboral. Por su parte, la empresa escogerá aquel plan de pensiones que, operando durante un mismo horizonte temporal y teniendo el mismo valor actual los costes que con- 
llevan ambos tipos de planes (condición de indiferencia para la empresa) maximice la utilidad del trabajador (Bodie et al., 1992).

De esta forma y a través de la resolución del modelo de optimización intertemporal planteado y del posterior análisis, se trata de comprobar la incidencia que una oferta laboral flexible tiene sobre el bienestar de cada trabajador en términos de la utilidad reportada por el consumo y el ocio durante todo el horizonte temporal de vigencia de ambos planes, cuando se tienen en cuenta el efecto de la riqueza financiera, la evolución de los salarios (rentas de trabajo) y los tantos de rendimiento de las inversiones (Fischer, 1983; Hubbard, 1987). Para ello, se considerará que el plan de pensiones de prestación definida garantiza a cada trabajador una prestación de jubilación equivalente al salario que se estima en el último año de su actividad profesional. Por el contrario, en un plan de aportación definida la empresa promotora únicamente garantiza las aportaciones que anualmente dota a la cuenta de posición que el trabajador tiene en el fondo de pensiones, que consisten en un porcentaje establecido del salario que se devenga anualmente a favor del partícipe-trabajador en tanto permanezca activo (Bodie et al.,1992; Bone y Mitchel, 1997).

\subsection{Hipótesis del modelo}

Para analizar este modelo se establecen a continuación una serie de hipótesis de partida que están relacionadas con las variables y parámetros que intervienen en los dos tipos de planes de pensiones que analiza este trabajo:

Se considera la existencia de mercados incompletos, de lo que se deduce que puede existir incertidumbre tanto en los salarios a percibir por cada partícipetrabajador como en los tantos de rendimiento de las inversiones de los recursos financieros del fondo de pensiones (Bodie et al., 1988). Si los mercados fueran completos en el sentido expuesto por Arrow-Debreu, la elección del tipo de plan de pensiones sería irrelevante, puesto que en este caso, el partícipe-trabajador podría hacer uso de títulos-valores para conseguir una posición óptima (Sundaresan y Zapatero, 1991; Varian, 1998).

Se considera que los tantos de rendimiento de las inversiones del fondo del plan de pensiones y los tantos de interés a los que se actualizan o capitalizan las distintas funciones que intervienen en la valoración del plan coinciden en cada uno de los períodos del horizonte temporal planteado.

La incertidumbre salarial se supone específica de cada partícipe-trabajador y diversificable para la empresa promotora del plan de pensiones (Boyle et al., 1998; Panjer, 2006).

Se supone incorrelación entre los salarios, los rendimientos de las inversiones y los tantos de interés de actualización o capitalización utilizados en la valoración del plan. Por consiguiente, estas magnitudes son independientes entre sí. 
Cada partícipe-trabajador integrante del plan de pensiones se supone adverso al riesgo. Por este motivo, se elige para este análisis una función de utilidad de tipo logarítmica, creciente y cóncava, que verifica las condiciones que ha de cumplir la misma para que sea representativa de este tipo de inversor (Van Der Ploeg, 1989; Varian, 1998).

Los salarios que corresponden a cada trabajador van a considerarse, bien conocidos, o bien inciertos, lo cual va a repercutir en el cálculo de las prestaciones a percibir por los trabajadores en el momento de su jubilación. Éstas tomarán distintos valores según un caso u otro. Los salarios explícitos devengados a favor del trabajador en ambos tipos de planes son los mismos y, al comienzo del plan, por simplicidad, asumimos que $S_{0}=E_{0}\left(\tilde{S}_{j}\right)$ $\forall j=1,2, \ldots, T-1$.

\subsection{Variables y parámetros del modelo}

En este apartado se especifican y se definen las variables y los parámetros que caracterizan al modelo representativo de las dos modalidades de planes de pensiones objeto de estudio:

$\bar{C}=\left(C_{0}, C_{1}, \ldots, C_{T}\right)$ : Vector de consumos que corresponden a cada partícipe del plan durante los períodos de su actividad laboral en la empresa que lo promueve. Es una de las dos variable de control del problema planteado.

$\bar{L}=\left(L_{0}, \tilde{L}_{1}, \ldots, \tilde{L}_{T}\right)$ : Vector de horas de ocio utilizadas por cada partícipe del plan. Las horas dedicadas al ocio (tiempo libre) van a depender del total de tiempo posible de trabajo. Es la otra variable de control del problema.

$\bar{M}=\left(M_{0}, \tilde{M}_{1}, \ldots, \tilde{M}_{T}\right):$ Vector de recursos financieros o riqueza disponible por cada partícipe-trabajador del plan durante cada período del horizonte temporal considerado.

$\bar{i}=\left({ }_{0} i_{1}, \tilde{i}_{2}, \ldots,{ }_{T-1} \tilde{i}_{T}\right):$ Vector de tantos de interés técnicos de actualización o capitalización utilizados en valoración del plan en cada uno de los periodos del horizonte temporal analizado, con ${ }_{0} i_{1}$, el tanto de interés aplicado al intervalo $(0,1)$ y conocido en $t=0$. Los tantos de interés que rigen los siguientes períodos son desconocidos e inciertos hasta el comienzo de los mismos. En consecuencia, los factores de capitalización para estos periodos serán $R_{0}=1+{ }_{0} i_{1}$ y $\tilde{R}_{j}=1+{ }_{j} i_{j+1} \forall j=1,2, \ldots, T-1$. Por su parte, los factores de actualización o descuento sin riego para los distintos períodos del horizonte temporal del modelo son $V(0,1)=1 / R_{0}, V(0,2)=1 / R_{0} R_{1}, \ldots, V(0, T)=1 / R_{0} R_{1} \cdots R_{T-1}$. Cuando se 
da entrada al riesgo, las funciones de actualización para los distintos períodos del análisis son: $V(0,1)=1 / R_{0}, V(0,2)=1 / R_{0} E_{0}\left(\tilde{R}_{1}\right), \ldots, V(0, T)=1 / R_{0} E_{0}\left(\tilde{R}_{1}\right) \cdots E_{0}\left(\tilde{R}_{T-1}\right)$, donde el operador ${ }^{1} E_{0}$ representa las expectativas correspondientes al momento inicial.

$\bar{r}=\left({ }_{0} r_{1}, \tilde{r}_{2}, \ldots,{ }_{T-1} \tilde{r}_{T}\right):$ Vector de tantos de rendimiento de las inversiones de los activos del fondo del plan supuestos para el horizonte temporal objeto de análisis, siendo $Z_{0}=1+{ }_{0} r_{1}, \tilde{Z}_{1}=1+{ }_{1} \tilde{r}_{2}, \cdots, \tilde{Z}_{T-1}=1+{ }_{T-1} \tilde{r}_{T}$ los factores de capitalización o descuento correspondientes a los diferentes períodos del análisis para los rendimientos de las inversiones del fondo del plan. Cuando estos tantos de rendimiento no son conocidos sino inciertos, su evolución se describe por medio de un proceso markoviano de tipo discreto (Taha, 2007; Hillier y Lieberman, 2010).

$\bar{S}=\left({ }_{0} S_{1},{ }_{1} \tilde{s}_{2}, \ldots,{ }_{T-1} \tilde{s}_{T}\right)$ : Vector de tantos anuales de crecimiento salarial. Cuando su comportamiento es incierto, su evolución va a ser descrita a través de un proceso markoviano de tipo discreto similar al descrito para los tantos de rendimiento de las inversiones.

$\bar{S}=\left(S_{0}, \tilde{S}_{1}, \ldots, \tilde{S}_{T-1}\right):$ Vector de salarios por hora que se devengan a favor de cada partícipe-trabajador de la empresa promotora del plan durante cada período. Estos salarios pueden ser conocidos y constantes en todo momento, o también inciertos.

$K_{t}$ : Porcentaje que, sobre el salario del trabajador, se compromete a aportar anualmente la empresa a su favor en un plan de pensiones de aportación definida. Este porcentaje se define $\forall t=0,1, \ldots, T-1$, como:

$$
K_{0}=\frac{1}{T} V(0, T), K_{1}=\frac{1}{T}\left[\frac{V(0, T)}{V(0,1)}\right], \ldots, K_{T-1}=\frac{1}{T}\left[\frac{V(0, T)}{V(0, T-1)}\right] .
$$

Estos coeficientes de ponderación son iguales a $K_{t}=1 / T \forall t=0,1, \ldots, T-1$, de tal forma que su suma sea la unidad, con el objeto de garantizar la condición de indiferencia de la empresa, es decir, que el valor actual del coste de pensiones en ambos tipos de planes coincida.

$B_{T}$ : Prestación por jubilación pagadera al trabajador que adquiere la condición de pensionista en el momento $T$. Esta variable va a tomar diferentes valores según se trate de un plan de aportación definida o de uno de prestación definida.

\footnotetext{
${ }^{1}$ Este operador debe interpretarse como una esperanza matemática que va a depender de la información disponible en ese momento.
} 
${ }^{A D} B_{0}$ : Valor actual de las contribuciones anuales realizadas por la empresa al plan de aportación definida. Teniendo en cuenta la hipótesis del modelo de que $S_{0}=E_{0}\left\{\tilde{S}_{1}\right\}=\cdots=E_{0}\left\{\tilde{S}_{T-1}\right\}$, se define así esta función:

$$
\begin{aligned}
{ }^{A D} B_{0} & =K_{0} S_{0}+K_{1} E_{0}\left\{\tilde{S}_{1}\right\} V(0,1)+\cdots+K_{T-1} E_{0}\left\{\tilde{S}_{T-1}\right\} V(0, T-1) \\
& =\frac{1}{T} V(0, T) S_{0}+\frac{1}{T}\left(\frac{V(0, T)}{V(0,1)}\right) S_{0} V(0,1)+\ldots+\frac{1}{T}\left(\frac{V(0, T)}{V(0, T-1)}\right) S_{0} V(0, T-1)=S_{0} V(0, T) .
\end{aligned}
$$

${ }^{P D} B_{0}$ : Valor actual de las prestaciones proyectadas de pensión de jubilación para cada partícipe del plan de prestación definida. Considerando los factores de descuento y de rendimiento de las inversiones, esta función se define así:

$$
{ }^{P D} B_{0}=E_{0}\left\{\tilde{S}_{T-1}\right\} V(0, T)=S_{0} V(0, T) .
$$

${ }^{A D} B_{T}$ : Valor final, a la edad de jubilación, de las prestaciones que corresponden a cada partícipe del plan de aportación definida. Esta función está definida por la expresión:

$$
\begin{aligned}
{ }^{P D} B_{T}= & \frac{1}{T} V(0, T) S_{0} Z_{0} E_{0}\left(Z_{1}\right) \cdots E_{0}\left(Z_{T-1}\right)+\frac{1}{T}\left(\frac{V(0, T)}{V(0,1)}\right) S_{1} Z_{1} E_{0}\left(Z_{2}\right) \cdots E_{0}\left(Z_{T-1}\right) \\
& +\ldots+\frac{1}{T}\left(\frac{V(0, T)}{V(0, T-1)}\right) S_{T-1} Z_{T-1} .
\end{aligned}
$$

En esta expresión, si los tantos de rendimiento de las inversiones coinciden con los tantos de descuento o de actualización financiera aplicados, y se consideran las demás hipótesis planteadas, la expresión anterior se traduce a:

$$
{ }^{A D} B_{T}=\frac{1}{T}\left(S_{0}+\tilde{S}_{1}+\cdots+\tilde{S}_{T-1}\right) .
$$

${ }^{P D} B_{T}$ : Valor final de las prestaciones proyectadas a la jubilación de cada partícipe del plan de prestación definida: ${ }^{P D} B_{T}=\tilde{S}_{T-1}$.

\subsection{Planteamiento del problema de optimización}

El análisis de los planes de pensiones de aportación y de prestación definida que se plantea en este trabajo se realiza en términos del bienestar que le reporta a cada trabajador el consumo y el ocio, considerando la condición de indiferencia para la empresa ya descrita. Para conseguir este objetivo, se plantea un mo- 
delo de programación dinámica ${ }^{2}$ para $T$ periodos anuales de tiempo, en el que las preferencias por el consumo y el ocio de cada partícipe del plan vienen representadas a través de una función de utilidad de tipo logarítmico aditiva a lo largo del tiempo (Deaton, 1995). Esta función creciente y cóncava, que describe adecuadamente la aversión al riesgo de cada uno de los trabajadores (Varian, 1998), viene dada por la expresión:

$$
U\left(C_{0}, \ldots, C_{T}, L_{0}, \ldots, L_{T-1}\right)=\ln \left(C_{0}\right)+E_{0}\left\{\sum_{n=1}^{T} \ln \left(C_{n}\right)\right\}+\ln \left(L_{0}\right)+E_{0}\left\{\sum_{n=1}^{T-1} \ln \left(L_{n}\right)\right\} .
$$

En el momento final del análisis, $T$, se supone que cada trabajador consumirá todos sus recursos disponibles, considerando entre ellos la prestación de jubilación que percibirá en ese momento. Esta prestación de jubilación va a coincidir con el salario al comienzo del último período del análisis. También se supone que en el último período todo el tiempo disponible del trabajador lo dedica al ocio, lo que implica que no se considere salario al final de dicho período. Por tanto, la riqueza financiera disponible en el momento final del análisis viene definida según la siguiente restricción presupuestaria:

$$
M_{T}=C_{T}=\left(M_{T-1}+S_{T-1}-C_{T-1}\right) R_{T-1}+B_{T} .
$$

Con este planteamiento, se resuelve recursivamente el problema de optimización intertemporal ${ }^{3}$ para $T$ periodos como una secuencia de problemas de optimización para dos períodos. Para ello se maximizará la función objetivo condicionada a la restricción presupuestaria de cada período, empezando por el final $(T-1, T)$ :

$$
\begin{gathered}
\operatorname{máx}_{C_{T-1}, C_{T}, L_{T-1}} U\left(C_{T-1}, C_{T}, L_{T-1}\right)=\ln \left(C_{T-1}\right)+\ln \left(C_{T}\right)+\ln \left(L_{T-1}\right) \\
\text { s.a: } \quad M_{T}=C_{T}=\left(M_{T-1}+S_{T-1}-C_{T-1}\right) R_{T-1}+B_{T} \\
\quad=\left(M_{T-1}+p\left(1+{ }_{T-2} s_{T-1}\right)\left(1-L_{T-1}\right)-C_{T-1}\right) R_{T-1}+p\left(1+{ }_{T-2} s_{T-1}\right)\left(1-L_{T-1}\right) .
\end{gathered}
$$

\section{SIMULACIÓN DEL MODELO}

Para poder obtener y comparar los valores que pueden tomar las distintas funciones que caracterizan al modelo y que intervienen en la valoración de un

\footnotetext{
${ }^{2}$ Para resolver el problema de optimización intertemporal utilizamos el método de la programación dinámica, que es una técnica que sirve para resolver problemas de optimización en los que existen diversos períodos de análisis descomponiéndolos en problemas de optimización para cada período (Varian, 1998; Barbolla et al., 2001).

${ }^{3}$ Para ambos tipos de planes de pensiones, la resolución de este problema de optimización intertemporal viene desarrollada en el Anexo al final de este trabajo.
} 
plan de pensiones de aportación o de prestación definida creados por la empresa a favor de sus trabajadores, se pasa a realizar una simulación práctica del modelo. Para este objetivo, se van a plantear dos posibles alternativas, según que los tantos de rendimiento de las inversiones del plan y de crecimiento de los salarios sean conocidos o inciertos. Para describir la evolución en el tiempo de estos tantos cuando no son conocidos, se utilizará un proceso markoviano de tipo discreto. De esta manera, se pasa a continuación a analizar estos dos casos:

\subsection{Tantos de rendimiento de las inversiones inciertos y tantos de creci- miento salarial conocidos}

En este primer caso, se considera que la evolución de los tantos de rendimiento de las inversiones del fondo del plan de pensiones es desconocida y, por tanto, incierta en el momento de la realización del contrato del plan de pensiones, mientras que la de los tantos de crecimiento de los salarios es conocida e independiente de los referidos tantos de rendimiento de las inversiones (coincidentes con los tantos de interés según hipótesis) y, por tanto, constantes en todo período.

Para modelizar la posible evolución de los tantos de rendimiento de las inversiones del fondo se emplea un proceso markoviano en tiempo discreto (Taha, 2007; Hilier y Lieberman, 2010). De esta manera, la matriz de transición que recoge la posible evolución entre los estados de la cadena de Markov (tantos de rendimiento anuales de las inversiones), en términos de probabilidades y, para el periodo genérico de tiempo anual $(t, t+1)$, se describe en la Tabla 1:

Tabla 1

Matriz de transición

\begin{tabular}{l|lll}
\hline $\mathbf{t} / \mathbf{t + 1}$ & $\boldsymbol{r}^{B}$ & $\boldsymbol{r}^{M}$ & $\boldsymbol{r}^{A}$ \\
\hline$r^{\beta}$ & $P_{B B}$ & $P_{B M}$ & $P_{B A}$ \\
$r^{M}$ & $P_{M B}$ & $P_{M M}$ & $P_{M A}$ \\
$r^{A}$ & $P_{A B}$ & $P_{A M}$ & $P_{A A}$ \\
\hline
\end{tabular}

Fuente: Elaboración propia.

Para este estudio, se supone que los tantos de rendimientos de las inversiones del fondo de pensiones pueden tomar tres valores posibles: bajo, $r^{B}$, medio, $r^{M}, \mathrm{y}$ alto, $r^{A}$ y que las probabilidades ${ }^{4}$ de evolución entre $(t, t+1)$ de los

\footnotetext{
${ }^{4}$ Estas probabilidades, de carácter subjetivo, se han establecido considerando que en el momento actual de crisis económica y financiera existe de un período a otro mayor probabilidad de mantenerse en el mismo tanto de rendimiento cuanto más bajo sea éste y que, a su vez, es más probable la evolución a tantos de rendimiento más bajos que a tantos de rendimientos más altos.
} 
estados del proceso vienen descritas a través de la matriz de transición entre estados $P$ :

$$
P=\left(\begin{array}{lll}
P_{B B} & P_{B M} & P_{B A} \\
P_{M B} & P_{M M} & P_{M A} \\
P_{A B} & P_{A M} & P_{A A}
\end{array}\right)=\left(\begin{array}{lll}
0,60 & 0,30 & 0,10 \\
0,50 & 0,40 & 0,10 \\
0,10 & 0,60 & 0,30
\end{array}\right) .
$$

También se supone que en el momento inicial los tantos de rendimiento de las inversiones que se pueden obtener en el mercado pueden tomar los valo$\operatorname{res}\left(\begin{array}{lll}r_{0}^{B} & r_{0}^{M} & r_{0}^{A}\end{array}\right)=\left(\begin{array}{lll}0,04 & 0,05 & 0,06\end{array}\right)$ según el riesgo que conlleven dichas inversiones y que el vector de probabilidades iniciales para dichos tantos es $\left(\begin{array}{lll}a_{B}^{0} & a_{M}^{0} & a_{A}^{0}\end{array}\right)=\left(\begin{array}{lll}0 & 1 & 0\end{array}\right)$, lo que equivale a decir, que en el momento inicial del estudio se considera que el tanto de rendimiento que rige en el mercado de capitales es el medio, es decir, que $r_{0}^{M}=0,05$. De esta manera, se determinan los tantos de rendimiento medio de las inversiones del fondo de pensiones estimados para cada periodo (media ponderada de los valores que pueden tomar por las probabilidades con que los toman):

$$
\begin{aligned}
& { }_{0} r_{1}={ }_{0} r_{1}^{B} P_{M B}+{ }_{0} r_{1}^{M} P_{M M}+{ }_{0} r_{1}^{A} P_{M A}=0,0460, \\
& { }_{1} r_{2}={ }_{0} r_{1}^{B} P_{M B}^{2}+{ }_{0} r_{1}^{M} P_{M M}^{2}+{ }_{0} r_{1} P_{M A}^{2}=0,0461 .
\end{aligned}
$$

Una vez establecida la ley que determina la evolución de los rendimientos de las inversiones del fondo del plan, se pasa a determinar el bienestar, en términos de consumo y de ocio, que reporta a cada partícipe-trabajador el plan de pensiones constituido por la empresa, tanto cuando se trata de un plan de prestación definida como de aportación definida. Para ello, es necesario conocer los factores de capitalización anuales para cada uno de los tres períodos del análisis:

$$
\begin{aligned}
& R_{0}=\left(1+{ }_{0} i_{1}\right)=\left(1+{ }_{0} r_{1}\right)=1,05 ; R_{1}=\left(1+{ }_{1} r_{2}\right)=1,046 ; R_{2}=\left(1+{ }_{2} r_{3}\right)=1,0461 ; \\
& a=\left(1+{ }_{0} s_{1}\right)=\left(1+{ }_{T-3} s_{T-2}\right)=1 ; b=\left(1+{ }_{1} s_{2}\right)=\left(1+{ }_{T-2} s_{T-1}\right)=1 ; S_{t}=p=40 u \text { u.m. } \forall t ; M_{0}=100 u . m .
\end{aligned}
$$

Los resultados obtenidos de la simulación del modelo para estos valores se recogen a continuación en la Tabla 2 y en las Figuras 1, 2, 3 y 4. De esta manera, se obtienen los valores óptimos de consumo, ocio, riqueza financiera y utilidad indirecta para el caso particular de este análisis de tres periodos, con un salario por hora inicial de 40 u.m. (unidades monetarias) y una riqueza financiera inicial de 100 u.m. 
Tabla 2

Valores óptimos de las variables del modelo

\begin{tabular}{|c|c|c|c|c|c|c|c|c|}
\hline \multirow{2}{*}{} & \multicolumn{4}{|c|}{ APORTACIÓN DEFINIDA } & \multicolumn{4}{c|}{ PRESTACIÓN DEFINIDA } \\
\cline { 2 - 9 } & $C_{t}^{*}$ & $L_{t}^{*}$ & $M_{t}$ & $V_{t}\left(M_{t}\right)$ & $C_{t}^{*}$ & $L_{t}^{*}$ & $M_{t}$ & $V_{t}\left(M_{t}\right)$ \\
\hline$t=0$ & 35,619 & 0,690 & 100 & 13,570 & 35,619 & 0,890 & 100 & 13,697 \\
\hline$t=1$ & 37,400 & 0,717 & 80,611 & 10,368 & 37,400 & 0,935 & 72,201 & 10,240 \\
\hline$t=2$ & 39,120 & 0,742 & 57,054 & 7,079 & 39,120 & 0,500 & 39,122 & 6,685 \\
\hline$t=3$ & 40,923 & 1 & 40,923 & 3,712 & 40,923 & 1 & 40,923 & 3,712 \\
\hline
\end{tabular}

Fuente: Elaboración propia.

Figura 1

Consumo

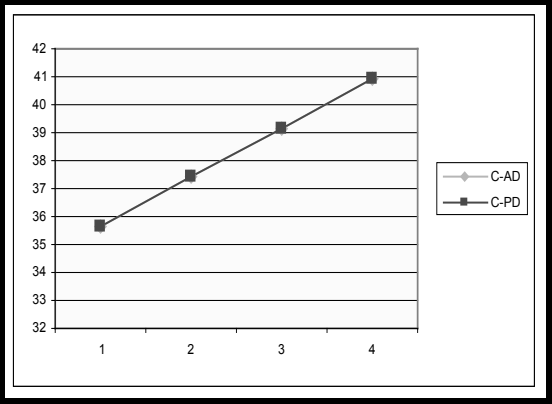

Fuente: Elaboración propia.

Figura 3

Riqueza

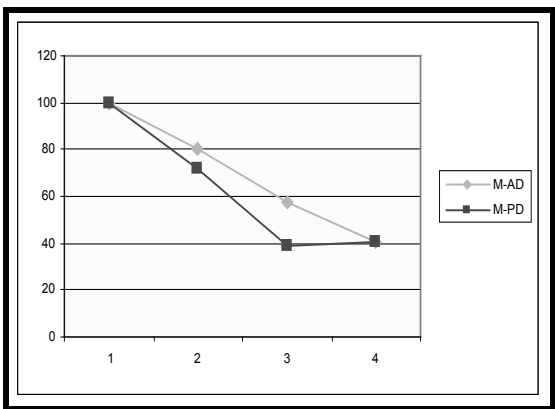

Fuente: Elaboración propia.
Figura 2

Ocio

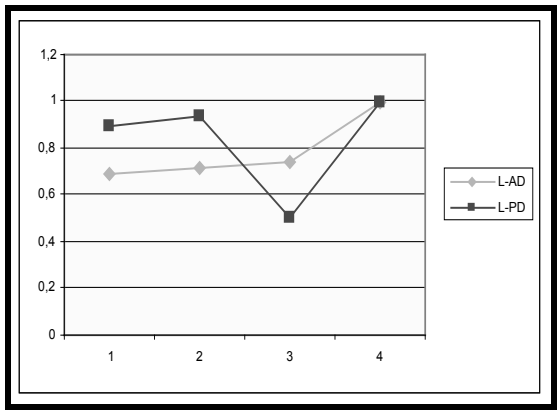

Fuente: Elaboración propia

Figura 4

Utilidad indirecta

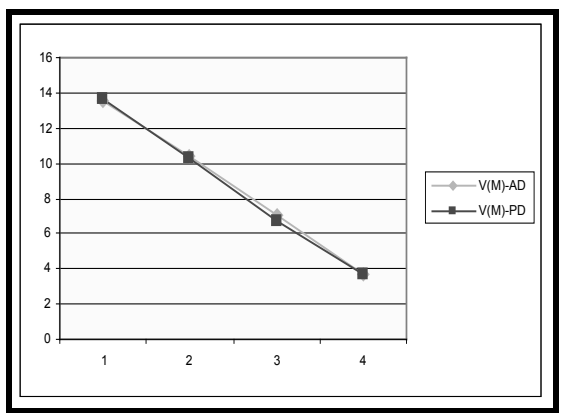

Fuente: Elaboración propia. 


\subsection{Tantos de rendimiento de las inversiones y tantos de crecimiento salarial inciertos}

En este apartado se considera que los tantos de rendimiento de las inversiones y los de crecimiento salarial, y, en consecuencia, los salarios, son desconocidos e inciertos en el momento de la implantación del plan, a la vez que incorrelacionados. Para poder realizar un análisis comparativo, se supone que la evolución de ambos se recoge a través de procesos markovianos en tiempo finito y con un número concreto de estados. En este caso, para describir la misma se definen de forma independiente dos matrices que recogerán, respectivamente, las probabilidades de transición en el tiempo de los tantos anuales de crecimiento salarial y de los rendimientos de las inversiones del fondo del plan, siendo la evolución de los tantos de rendimiento, y, por tanto, la matriz de paso entre estados, la misma que para el caso anterior.

Se supone que los tantos anuales de crecimiento de los salarios pueden tomar tres valores: bajo, $s^{B}$, medio, $s^{M}$, y alto, $s^{A}$, con distintas probabilidades, que se describen, a continuación, en la Tabla 3.

Tabla 3

Matriz de transición

\begin{tabular}{l|lll}
\hline $\mathbf{t} / \mathbf{t + 1}$ & $\boldsymbol{s}^{B}$ & $\boldsymbol{s}^{M}$ & $\boldsymbol{s}^{A}$ \\
\hline$s^{B}$ & $P_{B B}^{\prime}$ & $P_{B B}^{\prime}$ & $P_{B A}^{\prime}$ \\
$s^{M}$ & $P_{M B}^{\prime}$ & $P_{M M}^{\prime}$ & $P_{M A}^{\prime}$ \\
$s^{A}$ & $P_{A B}^{\prime}$ & $P_{A M}^{\prime}$ & $P_{A A}^{\prime}$ \\
\hline
\end{tabular}

Fuente: Elaboración propia.

Para este caso, se define el vector de valores iniciales conocidos para los tantos de crecimiento de los salarios $\left(\begin{array}{lll}s_{0}^{B} & s_{0}^{M} s_{0}^{A}\end{array}\right)=\left(\begin{array}{lll}0,015 & 0,020 & 0,030\end{array}\right)$; el vector de probabilidades iniciales para dichos tantos $\left(\begin{array}{lll}a_{B}^{\prime 0} & a_{M}^{\prime 0} & a_{A}^{\prime 0}\end{array}\right)=\left(\begin{array}{lll}0 & 1 & 0\end{array}\right)$, esto es, que en el momento inicial del estudio el tanto de crecimiento de los salarios que rige en el sector empresarial en el que se desarrolla el plan de pensiones es el bajo, es decir, que $s_{0}^{B}=0,015$, y la matriz $P^{\prime}$ recoge la evolución de los tantos de crecimiento salarial a través de un nuevo proceso markoviano de tipo discreto, en término de probabilidades ${ }^{5}$, para el periodo $(t, t+1)$ :

\footnotetext{
${ }^{5}$ Estas probabilidades, de carácter subjetivo, se consideran, en este caso, que se mantienen y tienden a crecer o decrecer para el tanto de crecimiento salarial bajo y para el medio, respectivamente.
} 


$$
P^{\prime}=\left(\begin{array}{lll}
P_{B B}^{\prime} & P_{B M}^{\prime} & P_{B A}^{\prime} \\
P_{M B}^{\prime} & P_{M M}^{\prime} & P_{M A}^{\prime} \\
P_{A B}^{\prime} & P_{A M}^{\prime} & P_{A A}^{\prime}
\end{array}\right)=\left(\begin{array}{ccc}
0,40 & 0,60 & 0,00 \\
0,00 & 0,90 & 0,10 \\
0,00 & 0,00 & 1,00
\end{array}\right) .
$$

Con estos datos, a continuación se determinan los tantos de crecimiento medio de los salarios estimados para cada periodo (media ponderada de los valores que toman por las probabilidades con que toman dichos valores). Para el caso concreto de tres períodos, se tiene:

$$
\begin{aligned}
& { }_{0} s_{1}={ }_{0} s_{1}^{B} P_{M B}^{\prime}+{ }_{0} s_{1}^{M} P_{M M}^{\prime}+{ }_{0} s_{1}^{A} P_{M A}^{\prime}=0,015, \\
& { }_{1} s_{2}={ }_{0} s_{1}^{B} P_{M B}^{\prime 2}+{ }_{0} s_{1}^{M} P_{M M}^{\prime 2}+{ }_{0} s_{1} P_{M A}^{\prime 2}=0,018 .
\end{aligned}
$$

De forma similar a la del caso anterior, se determinan los factores de capitalización de cada período para poder realizar una aplicación real del modelo:

$$
R_{0}=\left(1+{ }_{0} i_{1}\right)=\left(1+{ }_{0} r_{1}\right)=1,05 ; R_{1}=\left(1+{ }_{1} r_{2}\right)=1,046 ; R_{2}=\left(1+{ }_{2} r_{3}\right)=1,0461 \text {. }
$$

Del mismo modo, los valores finales de la prestación de jubilación para los planes de prestación definida y de aportación definida, respectivamente, son:

$$
\begin{gathered}
{ }^{P D} B_{T}=\tilde{S}_{T-1}=p\left(1+{ }_{T-2} S_{T-1}\right)\left(1-L_{T-1}\right), \\
{ }^{A D} B_{T}=\frac{1}{T}\left(S_{0}+\tilde{S}_{1}+\cdots+\tilde{S}_{T-1}\right)=\frac{p}{3}\left(\left(1-L_{T-1}\right)+a\left(1-L_{T-2}\right)+b\left(1-L_{T-3}\right)\right), \\
a=\left(1+{ }_{0} S_{1}\right)=\left(1+{ }_{T-3} S_{T-2}\right)=1,015 ; b=\left(1+{ }_{1} S_{2}\right)=\left(1+{ }_{T-2} S_{T-1}\right)=1,018 .
\end{gathered}
$$

De esta manera, se pasa a determinar los valores óptimos del consumo, ocio, riqueza y utilidad indirecta (Tabla 4 y Figuras $5,6,7,8$ ) para un caso particular en de tres periodos, con $T=3$, salario inicial $p=S_{0}=40 u . m$. (unidades monetarias) y la riqueza inicial, $M_{0}=100 u . m$.

\section{Tabla 4}

Valores óptimos de las variables del modelo

\begin{tabular}{|c|c|c|c|c|c|c|c|c|}
\hline \multirow{2}{*}{} & \multicolumn{4}{|c|}{ APORTACIÓN DEFINIDA } & \multicolumn{4}{c|}{ PRESTACIÓN DEFINIDA } \\
\cline { 2 - 9 } & $C_{t}^{*}$ & $L_{t}^{*}$ & $M_{t}$ & $V_{t}\left(M_{t}\right)$ & $C_{t}^{*}$ & $L_{t}^{*}$ & $M_{t}$ & $V_{t}\left(M_{t}\right)$ \\
\hline$t=0$ & 35,849 & 0,695 & 100 & 13,582 & 35,883 & 0,897 & 100 & 13,716 \\
\hline$t=1$ & 37,641 & 0,711 & 80,183 & 10,367 & 37,678 & 0,928 & 71,645 & 10,244 \\
\hline$t=2$ & 39,372 & 0,733 & 56,787 & 7,081 & 39,411 & 0,495 & 38,587 & 6,690 \\
\hline$t=3$ & 41,188 & 1 & 41,188 & 3,718 & 41,228 & 1 & 41,228 & 3,719 \\
\hline
\end{tabular}

Fuente: Elaboración propia. 
Figura 5

Consumo

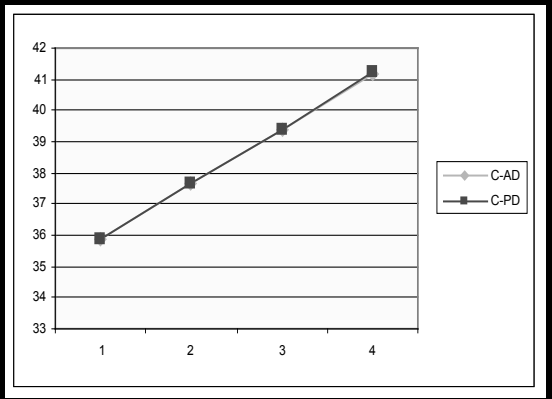

Fuente: Elaboración propia.

Figura 7

Riqueza

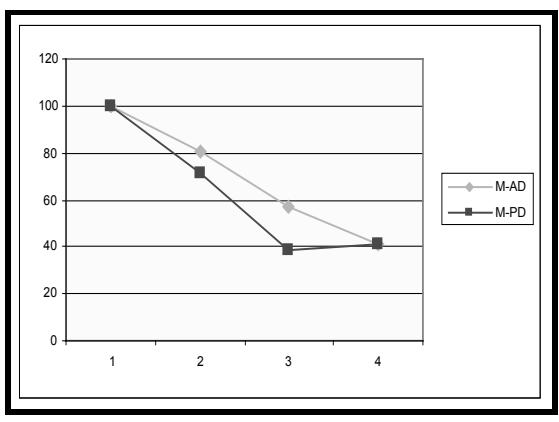

Fuente: Elaboración propia.
Figura 6

Ocio

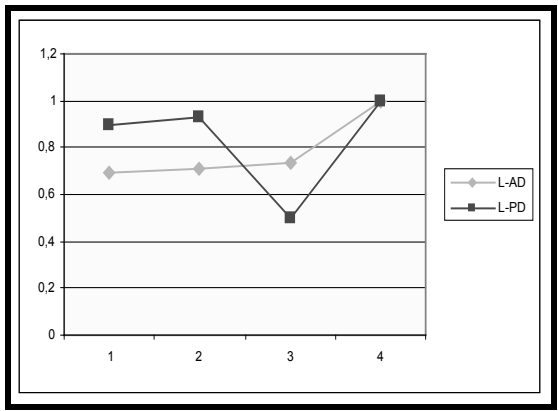

Fuente: Elaboración propia.

Figura 8

Utilidad indirecta

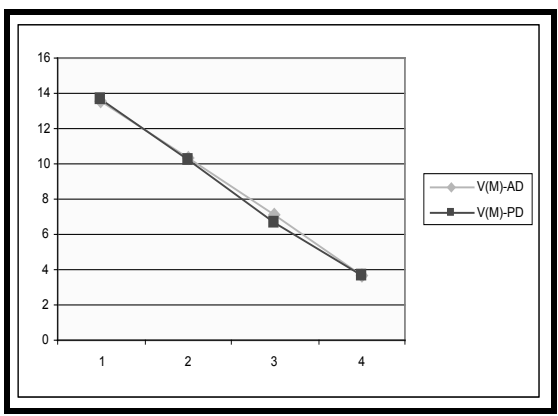

Fuente: Elaboración propia.

\section{CONCLUSIONES}

De los resultados obtenidos de la simulación del modelo para ambos tipos de planes de pensiones, teniendo en cuenta que se parte del mismo nivel de riqueza inicial, se deducen estos comentarios:

En el plan de pensiones de aportación definida, el número de horas dedicadas al ocio va aumentando a medida que transcurre el tiempo, mientras que en el plan de prestación definida este número aumenta y disminuye alternativamente. Cuando consideramos el caso de los tantos de rendimientos de las inversiones inciertos y los tantos de crecimiento de los salarios conocidos, los niveles de ocio óptimo en cada periodo tienen un valor superior al que alcanzan en el caso de tantos de rendimiento de las inversiones y de crecimiento salarial inciertos.

Para una misma riqueza inicial, los resultados obtenidos para el consumo óptimo coinciden, en el primer caso, siendo, en el segundo caso, ligeramente 
superiores para el plan de prestación definida. Por su parte, la riqueza financiera es superior para los planes de aportación definida en ambos casos, salvo al final del último periodo en el que prácticamente coinciden. En cuanto a la utilidad indirecta, salvo en el momento inicial, es mayor en ambos casos en los planes de aportación definida, excepto al final del último periodo en el que coincide en ambos.

Referente a los niveles de consumo óptimos, éstos son iguales en el plan de aportación definida y superiores en el primer caso donde los tantos de rendimientos de las inversiones son inciertos y los tantos de crecimiento de los salarios conocidos.

Si este análisis se hubiera realizado para mayores niveles de riqueza financiera inicial, el tiempo dedicado al ocio y el consumo aumentarían en ambos tipos de planes. Del mismo modo, a medida que creciera el salario de cada partícipe del plan, aumentaría el consumo y disminuiría el tiempo dedicado al ocio en cada período en ambos tipos de planes, disminuyendo la riqueza en el plan de prestación definida y aumentando en el plan de aportación definida.

\section{REFERENCIAS BIBLIOGRÁFICAS}

ALEXANDER, G.J. y SHARPE, W.F. (1989): Fundamentals of Investments. Nueva Jersey: Prentice-Hall.

BARBOLLA, R., CERDÁ, E. y SANZ, P. (2001): Optimización: Cuestiones, Ejercicios y Aplicaciones a la Economía. Madrid: Prentice Hall.

BONE, CH.M. y MITCHEL, O.S. (1997): Building better retirement income models". North American Actuarial Journal (NAAJ), Vol. 1, pp. 1-12.

BODIE, Z., MARCUS, A.J. y MERTON, R.C. (1988): "Defined benefit versus defined contribution pension plans: what are the real trade-offs?". Pensions in the U.S. Economy, pp. 139-162. Editado por Bodie, Z., Shoven, B.J. y Wise, A.D. Chicago: The University of Chicago Press.

BODIE, Z., MERTON, R.C. y SAMUELSON, W.F. (1992): "Labor supply flexibility and portfolio choice in a life cycle model". Journal of Economic Dynamics and Control, Vol. 16, pp. 427-449.

BOYLE, P.P., COX, S.H., DUFRESNE, D., GERBER, H.U., MUELLER, H.H., PEDERSEN, H.W., PLISKA, S.R., SHERRIS, M., SHIU, E.S. y TAN, K.S. (1998): Financial Economics: With Applications to Investments, Insurance and Pensions. Schaumburg, Illinois: The Actuarial Fundation.

DEATON, A. (1995): El Consumo. Madrid: Alianza Editorial.

DEATON, A. y MUELLBAUER, J. (1994): Economics and Consumer Behavior. Cambrigde University Press. Nueva York. 
FISCHER, S. (1983): "Investing for the short and the long term". Financial Aspects of the United States Pension System, pp. 153-156. Editado por Bodie, Z. y Shoven, B.J. Chicago: The University of Chicago Press.

HECKMAN, J.J. (1974): "Life-cycle comsuption and labor supply: an exploration of the relationship between income and consumption over the life cycle". American Economic Review, Vol. 64, pp. 188-194.

HILLIER, F.S. y LIEBERMAN, G.J. (2010): Introducción a la Investigación de Operaciones. México: MacGraw-Hill.

HUBBARD, R.G. (1987): "Uncertain lifetimes, pensions, and individual saving". Pensions in the U.S. Economy, pp. 175-210. Editado por Bodie, Z., Shoven, B.J. y Wise, A.D. Chicago: The University of Chicago Press.

PANJER, H.H. (2006): Operational Risk: Modeling Analystics. Hoboken: John Wiley \& Sons.

PELÁEZ FERMOSO, F.J. y GARCÍA GONZÁleZ, A. (2004): Los Planes y Fondos de Pensiones: Un Análisis Financiero-Actuarial. Valladolid: Secretariado de Publicaciones e Intercambio Editorial de la Universidad de Valladolid.

PEÑA ESTEBAN, DE LA J.I. (2000): Planes de Previsión Social. Madrid: Editorial Pirámide.

SUNDARESAN, S. y ZAPATERO, F. (1991): "Valuation, optimal assets allocation and retirement incentives od pension plans". New York: Working Paper. Graduate School of Business. Columbia University.

TAHA, H.A. (2007): Operations Research: An Introduction. New Yersey: Pearson.

VAN DER PLOEG, F. (1989): Risk Aversion, Intertemporal Subtitution and Consumption: The CARA-LQ Problem. Center for Economic Research. Países Bajos: Universidad de Tilburg.

VARIAN, H.R. (2007): Microeconomía Intermedia: Un Enfoque Actual. BarceIona: Antoni Bosch.

VARIAN, H.R. (1998): Análisis Microeconómico. Barcelona: Antoni Bosch. 


\section{Anexo}

El problema de optimización intertemporal se resuelve por el método de los multiplicadores de Lagrange con el fin de determinar los niveles de consumo y ocio óptimos que corresponden a cada uno de los tres períodos anuales del horizonte temporal analizado. Este método se caracteriza porque, en primer lugar, se procede a determinar el consumo y el ocio óptimos para el último periodo ( $T-1$, T):

$$
\begin{gathered}
\underset{C_{T-1}, C_{T}, L_{T-1}}{\max } U\left(C_{T-1}, C_{T}, L_{T-1}\right)=\ln \left(C_{T-1}\right)+\ln \left(C_{T}\right)+\ln \left(L_{T-1}\right) \\
\text { s.a: } \quad M_{T}=C_{T}=\left(M_{T-1}+p b\left(1-L_{T-1}\right)-C_{T-1}\right) R_{T-1}+p b\left(1-L_{T-1}\right) .
\end{gathered}
$$

La función Lagrangiana asociada a este problema de optimización viene dada por:

$$
\begin{aligned}
& L\left(C_{T-1}, C_{T}, L_{T-1}, \lambda\right)=\ln \left(C_{T-1}\right)+\ln \left(C_{T}\right)+\ln \left(L_{T-1}\right) \\
& \quad-\lambda\left(C_{T}-\left(\left(M_{T-1}+p b\left(1-L_{T-1}\right)-C_{T-1}\right) R_{T-1}+p b\left(1-L_{T-1}\right)\right)\right),
\end{aligned}
$$

donde $\lambda$ es el multiplicador de Lagrange ${ }^{6}$ correspondiente a la restricción asociada a la riqueza financiera del trabajador al final de cada período. De esta manera, diferenciando la función Lagrangiana respecto de $C_{T-1,} L_{T-1}$ y $\lambda$, es decir, teniendo en cuenta las condiciones de óptimo:

$$
\begin{aligned}
\frac{\partial L}{\partial C_{T-1}} & =\frac{1}{C_{T-1}}-\lambda R_{T-1}=0, \\
\frac{\partial L}{\partial C_{T}} & =\frac{1}{C_{T}}-\lambda=0, \\
\frac{\partial L}{\partial L_{T-1}} & =\frac{1}{L_{T-1}}-\lambda p b\left(R_{T-1}+1\right)=0, \\
\frac{\partial L}{\partial \lambda} & =C_{T}-\left(\left(M_{T-1}+p b\left(1-L_{T-1}\right)-C_{T-1}\right) R_{T-1}+p b\left(1-L_{T-1}\right)\right)=0,
\end{aligned}
$$

se obtienen el consumo y ocio óptimos del último período del análisis:

\footnotetext{
${ }^{6}$ El multiplicador de Lagrange cambiado de signo representa la tasa marginal de cambio del valor óptimo de la función objetivo ante una variación en el término independiente de cada restricción, en este caso, la riqueza financiera. Ello justifica el nombre de "valor sombra de la restricción" con el que se suele también conocer a los multiplicadores de Lagrange (Barbolla et al., 2001).
} 


$$
\begin{aligned}
& C_{T}^{*}=R_{T-1} C_{T-1} \\
& L_{T-1}^{*}=\frac{1}{\lambda p b\left(R_{T-1}+1\right)}=\frac{C_{T-1} R_{T-1}}{p b\left(R_{T-1}+1\right)}
\end{aligned}
$$

Operando de forma similar tanto cuando se analiza un plan de prestación definida como uno de aportación definida, se obtienen los consumos y ocio óptimos para el periodo $(T-1, T)$ :

$$
\begin{gathered}
{ }^{P D} C_{T}^{*}=\frac{M_{T-1} R_{T-1}+p b\left(R_{T-1}+1\right)}{3} \\
{ }^{P D} C_{T-1}^{*}=\frac{M_{T-1} R_{T-1}+p b\left(R_{T-1}+1\right)}{3 R_{T-1}} \\
{ }^{P D} L_{T-1}^{*}=\frac{M_{T-1} R_{T-1}+p b\left(R_{T-1}+1\right)}{3 p b\left(R_{T-1}+1\right)} \\
{ }^{A D} C_{T}^{*}=\frac{p\left(b\left(3 R_{T-1}+1\right)+a+1\right)+3 M_{T-1} R_{T-1}-p\left(L_{T-3}+a L_{T-2}\right)}{9} \\
{ }^{A D} C_{T-1}^{*}=\frac{p\left(b\left(3 R_{T-1}+1\right)+a+1\right)+3 M_{T-1} R_{T-1}-p\left(L_{T-3}+a L_{T-2}\right)}{9 R_{T-1}} \\
{ }^{A D} L_{T-1}^{*}=\frac{p\left(b\left(3 R_{T-1}+1\right)+a+1\right)+3 M_{T-1} R_{T-1}-p\left(L_{T-3}+a L_{T-2}\right)}{3 b p\left(3 R_{T-1}+1\right)} .
\end{gathered}
$$

Determinados los niveles de consumo y de ocio óptimos, sustituyendo estos valores en la función objetivo a maximizar, se obtiene la correspondiente función valor o función de utilidad indirecta ${ }^{7}$. Para los planes de prestación definida y de aportación definida viene dada, respectivamente, por estas expresiones:

$$
\begin{gathered}
{ }^{P D} V_{T}\left(M_{T}\right)=\ln \left({ }^{P D} C_{T}^{*}\right)=\ln \left(\frac{M_{T-1} R_{T-1}+p b\left(R_{T-1}+1\right)}{3}\right) . \\
{ }^{P D} V_{T-1}\left(M_{T-1}\right)=\ln \left({ }^{P D} C_{T-1}^{*}\right)+\ln \left({ }^{P D} C_{T}^{*}\right)+\ln \left({ }^{P D} L_{T-1}^{*}\right)=\ln \left(\frac{\left(M_{T-1} R_{T-1}+p b\left(R_{T-1}+1\right)\right)^{3}}{27 R_{T-1} p b\left(R_{T-1}+1\right)}\right) .
\end{gathered}
$$

\footnotetext{
${ }^{7}$ Esta función representa la utilidad máxima alcanzable por el trabajador en términos de bienestar y está relacionada con su riqueza disponible en el período. La función de utilidad indirecta es esencialmente, como su propio nombre indica, una utilidad indirecta e denota la utilidad máxima que se obtiene en función de la riqueza disponible del consumidor (Varian, 1998).
} 


$$
\begin{gathered}
{ }^{A D} V_{T}\left(M_{T}\right)=\ln \left({ }^{A D} C_{T}^{*}\right)=\ln \left(\frac{p\left(b\left(3 R_{T-1}+1\right)+a+1\right)+3 M_{T-1} R_{T-1}-p\left(L_{T-3}+a L_{T-2}\right)}{9}\right) . \\
{ }^{A D} V_{T-1}\left(M_{T-1}\right)=\ln \left({ }^{A D} C_{T-1}^{*}\right)+\log \left({ }^{A D} C_{T}^{*}\right)+\log \left({ }^{A D} L_{T-1}^{*}\right) \\
=\ln \left(\frac{\left(p\left(b\left(3 R_{T-1}+1\right)+a+1\right)+3 M_{T-1} R_{T-1}-p\left(L_{T-3}+a L_{T-2}\right)\right)^{3}}{243 b p R_{T-1}\left(3 R_{T-1}+1\right)}\right) .
\end{gathered}
$$

Para calcular los niveles de consumo y ocio óptimos de los períodos $(T-2, T-1)$ y $(T-3, T-2)$ se procede de igual forma. Así, si el trabajador decide consumir de ambos bienes al comienzo del periodo $(T-2, T-1), C_{T-2}^{*}$ y $L_{T-2}^{*}$, la riqueza financiera al final será:

$$
M_{T-1}=\left(M_{T-2}+p a\left(1-L_{T-2}^{*}\right)-C_{T-2}^{*}\right) R_{T-2} .
$$

De esta manera, se determinan los niveles de consumo y de ocio óptimos correspondientes al periodo $(T-2, T-1)$, es decir, $C_{T-2}^{*}$ y $L_{T-2}^{*}$ :

$$
\begin{gathered}
{ }^{P D} C_{T-2}^{*}=\frac{M_{T-2} R_{T-2} R_{T-1}+p\left(R_{T-2} R_{T-1} a+b\left(R_{T-1}+1\right)\right)}{5 R_{T-2} R_{T-1}} \\
{ }^{P D} L_{T-2}^{*}=\frac{M_{T-2} R_{T-2} R_{T-1}+p\left(R_{T-2} R_{T-1} a+b\left(R_{T-1}+1\right)\right)}{5 a p R_{T-2} R_{T-1}} . \\
{ }^{A D} C_{T-2}^{*}=\frac{p\left(3 R_{T-1}\left(a R_{T-2}+b\right)+a+b+1\right)+3 M_{T-2} R_{T-2} R_{T-1}-p L_{T-3}}{15 R_{T-2} R_{T-1}} \\
{ }^{A D} L_{T-2}^{*}=\frac{p\left(3 R_{T-1}\left(a R_{T-2}+b\right)+a+b+1\right)+3 M_{T-2} R_{T-2} R_{T-1}-p L_{T-3}}{5 a p\left(3 R_{T-2} R_{T-1}+1\right)} .
\end{gathered}
$$

La utilidad que en términos de consumo y ocio le reportará a cada trabajador, vendrá dada por la función de utilidad indirecta, $V_{T-2}\left(M_{T-2}\right)$ :

$$
V_{T-2}\left(M_{T-2}\right)=\operatorname{máx}\left\{\ln \left(C_{T-2}^{*}\right)+\ln \left(L_{T-2}^{*}\right)+E_{T-2}\left(V_{T-1}\left(M_{T-1}\right)\right)\right\} \text {. }
$$

Sustituyendo en esta expresión el valor de la utilidad indirecta, $V_{T-1}\left(M_{T-1}\right)$, y los consumos y ocio óptimos calculados para este período, se determina la función de utilidad indirecta: 


$$
\begin{gathered}
{ }^{P D} V_{T-2}\left(M_{T-2}\right)=\ln \left(\frac{\left(R_{T-2} R_{T-1}\right)^{3}\left(\left(M_{T-2}+p a+\frac{p b\left(R_{T-1}+1\right)}{R_{T-2} R_{T-1}}\right) / 5\right)^{5}}{R_{T-1} p^{2} a b\left(R_{T-1}+1\right)}\right) \\
{ }^{A D} V_{T-2}\left(M_{T-2}\right)=\ln \left(\frac{\left(p\left(3 R_{T-1}\left(a R_{T-2}+b\right)+a+b+1\right)+3 M_{T-1} R_{T-2} R_{T-1}-p L_{T-3}\right)^{5}}{84375 R_{T-2} R_{T-1}^{2} a b p^{2}\left(3 R_{T-1}+1\right)\left(3 R_{T-2} R_{T-1}+1\right)}\right) .
\end{gathered}
$$

Por último, se determinan los valores del consumo y ocio óptimos para el primer periodo $(T-3, T-2)$. Así, si el trabajador decide consumir de ambos bienes al comienzo de este periodo $C_{T-3}^{*}$ y $L_{T-3}^{*}$, la riqueza al final del mismo será:

$$
M_{T-2}=\left(M_{T-3}+p\left(1-L_{T-3}^{*}\right)-C_{T-3}^{*}\right) R_{T-3} .
$$

Procediendo de igual forma que para los períodos anteriores, se tiene:

$$
\begin{aligned}
{ }^{P D} C_{T-3}^{*} & =\frac{M_{T-3} R_{T-3} R_{T-2} R_{T-1}+p\left(R_{T-3} R_{T-2} R_{T-1}+R_{T-2} R_{T-1} a+b\left(R_{T-1}+1\right)\right)}{7 R_{T-3} R_{T-2} R_{T-1}} \\
{ }^{P D} L_{T-3}^{*} & =\frac{M_{T-3} R_{T-3} R_{T-2} R_{T-1}+p\left(R_{T-3} R_{T-2} R_{T-1}+R_{T-2} R_{T-1} a+b\left(R_{T-1}+1\right)\right)}{7 p R_{T-3} R_{T-2} R_{T-1}} . \\
{ }^{A D} C_{T-3}^{*} & =\frac{p\left(3 R_{T-1}\left(R_{T-3} R_{T-2}+a R_{T-2}+b\right)+a+b+1\right)+3 M_{T-1} R_{T-3} R_{T-2} R_{T-1}}{21 R_{T-3} R_{T-2} R_{T-1}} \\
{ }^{A D} L_{T-3}^{*} & =\frac{p\left(3 R_{T-1}\left(R_{T-3} R_{T-2}+a R_{T-2}+b\right)+a+b+1\right)+3 M_{T-1} R_{T-3} R_{T-2} R_{T-1}}{7 p\left(3 R_{T-3} R_{T-2} R_{T-1}+1\right)} .
\end{aligned}
$$

La utilidad indirecta dada por la función $V_{T-3}\left(M_{T-3}\right)$ para este período es:

$$
\begin{gathered}
{ }^{P D} V_{T-3}\left(M_{T-3}\right)=\ln \left(\frac{\left(R_{T-2} R_{T-1}\right)^{3} R_{T-3}^{5}\left(\left(M_{T-3}+p+\frac{p a}{R_{T-3}}+\frac{p b\left(R_{T-1}+1\right)}{R_{T-3} R_{T-2} R_{T-1}}\right) / 7\right)^{7}}{R_{T-1} p^{3} a b\left(R_{T-1}+1\right)}\right) . \\
{ }^{A D} V_{T-3}\left(M_{T-3}\right)=\ln \left(\frac{\left(p\left(3 R_{T-1}\left(R_{T-3} R_{T-2}+a R_{T-2}+b\right)+a+b+1\right)+3 M_{T-1} R_{T-3} R_{T-2} R_{T-1}\right)^{7}}{66706983 R_{T-3} R_{T-2}^{2} R_{T-1}^{3} a b p^{3}\left(3 R_{T-1}+1\right)\left(3 R_{T-2} R_{T-1}+1\right)\left(3 R_{T-3} R_{T-2} R_{T-1}+1\right)}\right) .
\end{gathered}
$$


De esta forma, aplicando el método recursivo para el cálculo, podría determinarse, en general, la función de utilidad indirecta para cualquier momento $t \in\{0,1,2, \ldots, T\}$ del horizonte temporal que se desea analizar. 